\title{
Microtensile bond strength of CAD/CAM materials to dentin under different adhesive strategies. Braz Oral Res. 2017;31:e109.
}

Where is read:

Julieta Gomes TAVARES(c)

Ana Maria SPOHR ${ }^{(c)}$

(a) Pontifícia Universidade Católica da Universidade Federal do Rio Grande do Sul - PUC-RS, School of Dentistry, Graduate Program, Porto Alegre, RS, Brazil.

It should read:

Julieta Gomes TAVARES(a)

Ana Maria SPOHR ${ }^{(a)}$

(a)Pontifícia Universidade Católica do Rio Grande do Sul - PUC-RS, School of Dentistry, Porto Alegre, RS, Brazil.

Disconsider:

(c) Pontifícia Universidade Católica da Universidade Federal do Rio Grande do Sul - PUC-RS, School of Dentistry, Clinical Department, Porto Alegre, RS, Brazil. 